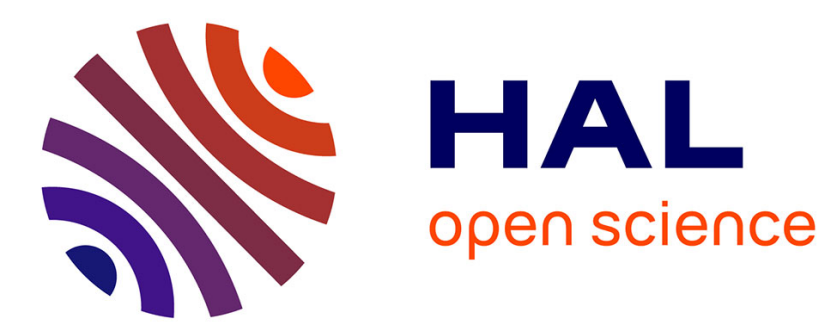

\title{
Sensibilité de 27 variétés de pêcher vis-à-vis de Fusicoccum amygdali Del., agent du chancre des rameaux
}

Francis Jailloux, Georges Froidefond

\section{To cite this version:}

Francis Jailloux, Georges Froidefond. Sensibilité de 27 variétés de pêcher vis-à-vis de Fusicoccum amygdali Del., agent du chancre des rameaux. Agronomie, 1985, 5 (6), pp.515-520. hal-00884779

\section{HAL Id: hal-00884779 \\ https://hal.science/hal-00884779}

Submitted on 1 Jan 1985

HAL is a multi-disciplinary open access archive for the deposit and dissemination of scientific research documents, whether they are published or not. The documents may come from teaching and research institutions in France or abroad, or from public or private research centers.
L'archive ouverte pluridisciplinaire HAL, est destinée au dépôt et à la diffusion de documents scientifiques de niveau recherche, publiés ou non, émanant des établissements d'enseignement et de recherche français ou étrangers, des laboratoires publics ou privés. 


\title{
Sensibilité de 27 variétés de pêcher vis-à-vis de Fusicoccum amygdali Del., agent du chancre des rameaux
}

\author{
Francis JAILLOUX \\ avec la collaboration technique de Georges FroIDEFOND
}

I.N.R.A., Station de Pathologie végétale, Centre de Recherches de Bordeaux, B.P. 131, F 33140 Pont-de-la-Maye

Le chancre du pêcher, dû à Fusicoccum amygdali Del., peut causer de graves dégâts dans certaines régions françaises telles que le Sud-Ouest. Dans ces conditions, la connaissance du niveau de sensibilité des variétés devient impérative.

Au cours de trois années successives, une collection de 27 variétés de pêcher sont contaminées artificiellement aux deux périodes de réceptivité de la plante : en automne par contamination de plaies pétiolaires provoquées $(1979,1980$ et 1981$)$ et au printemps par pollution des bourgeons entre les stades phénologiques D et $\mathrm{G}$ ( 1980 , 1981 et 1982).

Seule, la contamination des plaies pétiolaires provoquées permet de différencier des variétés :

- peu sensibles (« J. H. Hale », "I.N.R.A. $3689 »)$,

- très sensibles ( $«$ Shasta $», ~ «$ Redhaven $»)$,

- sensibles à comportement intermédiaire ( Fortyniner », « Frederica »).

La connaissance de la réceptivité variétale du pêcher à l'égard de $F$. amygdali of fre un critère supplémentaire de choix tant pour la création de futurs hybrides que pour l'implantation de nouveaux vergers.

Mots clés additionnels : Stade phénologique, bourgeon, plaie pétiolaire, dose d'inoculum, réceptivité

Peach canker disease is sometimes responsible for severe damage to peach orchards specially in South West France. In those areas which are favourable to disease development, it is important to know the level of resistance of different varieties before planting new orchards. During three years, susceptibility of 27 commercial cvs was tested at two periods known to be implicated in : inoculum at various concentrations was applied in autumn onto fresh leaf scars $(1979,80,81)$ and in spring onto buds (between $D$ and $G$ stages) $(1980,81,82)$. Varietal susceptibility as determined from the results of fresh leaf scar inoculation allows to classify the $27 \mathrm{cvs}$ into three classes of high ('Shasta', 'Redhaven'), moderate ('Fortyniner', Frederica') and low susceptibility ('J. H. Hale', 'I.N.R.A. 3689'). By contrast, no significant differences were observed when inoculum was applied to buds : in general, they appeared less susceptible than leaf scars which are undoubtedly the main sites of introduction of the fungus. It is of interest to know the level of susceptibility to peach canker of the different cvs both for establishing new plantations and for breeding against this pathogen.

Additional key words : Phenological stage, bud, leaf scar, inoculum concentration, receptivity.

\section{INTRODUCTION}

Dans les vergers de pêchers, on observe parfois de graves dégâts liés à la présence de chancres dus au champignon parasite Fusicoccum amygdali Del. Ces chancres qui s'extériorisent au cours des mois de mars, avril, mai, sont centrés sur des bourgeons (chancre de bourgeon) ou formés au point d'insertion des jeunes pousses herbacées sur les branches de l'année précédente (chancre de base). De tels dégâts sont généralement suivis de la mort des bourgeons ou du flétrissement plus ou moins rapide des jeunes pousSes (GROSCLAUDE, 1956).

La plupart des chancres produisent rapidement des organes de fructification ou "pycnides » exsudant de minuscules vrilles blanchâtres appelées «cirrhes", 
agglomérats de spores qui assurent de nouvelles contaminations et peuvent rester en activité plus de 3 ans en conditions naturelles, rendant la lutte chimique difficile dans les zones au climat particulièrement humide pendant les périodes de réceptivité (JAILlOUX \& FROIDEFOND, 1974).

$F$. amygdali étant essentiellement un parasite de blessure, la grande majorité des infections se réalise, en automne à la chute des feuilles, par les plaies pétiolaires et, au printemps, par les cicatrices formées par la chute des écailles et l'éclatement des bourgeons entre les stades $D$ (sortie des pétales) et $G$ (chute des pétales).

La connaissance du niveau de sensibilité des variétés (JAILLOUX \& FROIDEFOND, 1976, 1978a) doit orienter le choix de l'arboriculteur en cas de nouvelles plantations et la conduite d'une bonne protection sanitaire pour les plus sensibles. Le travail présenté consiste à comparer le niveau de sensibilité de 27 variétés de pêcher au cours de 3 années successives d'expérimentation sur le terrain.

\section{MATÉRIEL ET MÉTHODES}

\section{A. Le matériel végétal}

Une collection de 27 variétés greffées sur «GF 305 » est implantée en 1977 sur le domaine I.N.R.A. de Couhins à Villenave-d'Ornon (Gironde). Pour chaque variété, 4 arbres, répartis par blocs de 2, ont servi de base à l'étude.

\section{B. L'inoculum}

A partir de cultures pures du parasite sur milieu avoine gélosé $(40 \mathrm{~g} / \mathrm{l})$, âgées de 3 semaines, des suspensions aqueuses de spores sont ajustées aux concentrations désirées à l'aide d'une cellule hématimétrique de Malassez. Afin d'éliminer le facteur de variation dû à l'existence de races physiologiques éventuelles, l'inoculum est constitué d'un mélange de cultures issues d'isolements géographiques différents sur pêcher : Sainte-Livrade (Lot-et-Garonne), Lanxade (Dordogne), Couhins (Gironde), Castillon (Lot-etGaronne) et Manduel (Gard). Pour éviter la perte de pouvoir pathogène des différents isolats, les cultures conservées au réfrigérateur sont repiquées une fois par an.

\section{Méthodes de contamination}

Les contaminations sont réalisées aux 2 périodes de réceptivité du pêcher précisées ci-dessus (JAILLOUX \& FROIDEFOND, 1974) : en automne par inoculation de plaies pétiolaires provoquées $(1979,1980$ et 1981) et au printemps par apport d'une suspension de spores sur les bourgeons $(1980,1981$ et 1982) entre les stades phénologiques $\mathrm{D}$ et $\mathrm{G}$.

\section{Inoculation des plaies pétiolaires}

Les plaies pétiolaires sont obtenues par simple pression latérale sur le pétiole des feuilles pour entraîner la chute de ces dernières. Elles sont réalisées le 27 septembre en 1979 , le 18 septembre en 1980 et 1981, soit quelques jours avant le début de la défolia- tion naturelle. A l'aide d'une micro-seringue, une goutte $(1 / 50 \mathrm{ml})$ de suspension de spores est déposée aussitôt sur chaque plaie pétiolaire fraîche à raison de 50 inoculations par concentration d'inoculum et variété.

Afin d'éviter de masquer d'éventuelles différences variétales par une pression trop forte de la maladie (JAILLOUX \& FROIDEFOND, 1978b), nous utilisons 2 concentrations de spores assez faibles : 60 et 600 spores par plaie. En 1979 cependant, une seule concentration (600 spores par plaie) est utilisée.

\section{Contamination des bourgeons}

Les bourgeons sont contaminés, par dépôt d'une goutte d'une suspension de spores $(1 / 50 \mathrm{ml})$, les 18 mars 1980,17 mars 1981 et 5 mars 1982 ; 50 bourgeons par variété et par concentration sont ainsi traités, les variétés étant à des stades physiologiques comparables correspondant à la phase de sensibilité à la maladie (stade entre $D$ et $G$ ).

En raison des pertes d'inoculum liées à une mauvaise tenue des gouttes de suspension des spores à la surface de ces organes et de la réceptivité à la maladie, généralement assez faible sur les bourgeons (une faible production de filaments germinatifs parvenant à pénétrer dans le bourgeon), nous utilisons des doses d'inoculum plus élevées de 60000 et 600000 spores par bourgeon.

\section{Notation et analyse des résultats}

La dimension des chancres n'est pas retenue car des observations antérieures nous ont permis de constater qu'il n'y avait pas de corrélation entre leur longueur et la réceptivité variétale; les chancres peuvent être aussi développés sur des variétés peu réceptives que sur des variétés très sensibles, la résistance intervenant lors de la pénétration (PAYGHAMI, 1978) et non au niveau des tissus internes de l'hôte.

Pour les contaminations de plaies provoquées à l'automne, les notations sont réalisées régulièrement d'octobre à avril pour tenir compte des évolutions tardives des chancres. Elles consistent à comptabiliser les chancres qui se sont formés autour du coussinet pétiolaire inoculé.

Dans le cas des contaminations de bourgeons au printemps, les notations sont effectuées de mai à août, période où tous les chancres susceptibles de se former se sont extériorisés. Cela permet une notation qui tient compte de la formation de chancres au niveau des bourgeons infectés et à la base des jeunes pousses feuillées ayant pu évoluer à partir de ces bourgeons.

Les pourcentages d'infections suivant les variétés sont analysés statistiquement par le test non paramétrique de FRIEDMAN. On ordonne les variétés suivant leur sensibilité (la plus résistante ayant le rang 1) et on effectue la somme des rangs obtenus par variété, année et concentration d'inoculum. Les résultats acquis au cours des 3 années sont assimilables à des répétitions. Le test de FRIEDMAN utilise la formule suivante :

$\chi_{2}=\left(\frac{12}{N+K(K+1)} \times \varepsilon\right.$ chaque $\left.R^{2}{ }^{2}\right)-3 N(K+1)$ 
où :

$\mathrm{N}=$ nombre de traitements.

$\mathrm{K}=$ nombre de variétés.

$\mathrm{Rj}=$ pourcentage d'infections par variété pour les différents traitements.

Cette valeur est confrontée à une table de $\chi_{2}$ à ( $k$ - 1) degrés de liberté.

\section{RÉSULTATS ET DISCUSSION}

\section{A. Sensibilité des plaies pétiolaires suivant les variétés}

Les pourcentages d'inoculations positives obtenus chez les 27 variétés lors des 3 années d'expérimentation en fonction de chaque concentration d'inoculum permettent de classer ces variétés selon un ordre croissant de sensibilité si on tient compte de la moyenne générale (tabl. 1). L'analyse statistique des résultats met en évidence un effet hautement significatif de la concentration d'inoculum et de la réceptivité variétale.

Les différences entre les pourcentages d'inoculations positives étant très faibles pour certaines varié- tés, il a paru plus rationnel de regrouper les variétés dans 4 classes de sensibilité (tabl. 2) :

Classes $1=0-20$ p. 100 d'inoculations positives.

Classes $2=21-40$ p. 100 d'inoculations positives.

Classes $3=41-60$ p. 100 d'inoculations positives.

Classes $4=61-80$ p. 100 d'inoculations positives.

A partir des éléments du tableau 2 , le rôle de la pression d'inoculum sur la gravité des attaques apparaît clairement : à la concentration de 60 spores par plaie pétiolaire, 80 p. 100 des variétés présentent moins de 40 p. 100 d'inoculations positives alors qu'à la concentration de 600 spores, 60 p. 100 des variétés présentent plus de 60 p. 100 d'inoculations positives se traduisant par des chancres. On peut considérer que la dose de 60 spores ne permet pas d'assurer un criblage suffisant des variétés car certaines d'entre elles qui paraissent résistantes (classe 1) à 60 spores, peuvent se montrer très sensibles dès lors que la pression de maladie devient plus importante. C'est le cas de « Mme Girerd », « Cardinal », « Early O'Henry », qui, à 600 spores par plaie pétiolaire, sont placées dans la classe 4 .

TABLEAU 1

Comparaison de la réceptivité des plaies pétiolaires de 27 variétés de pêcher à Fusicoccum amygdali par inoculation artificielle d'automne sur 3 années.

Susceptibility to peach canker of 27 peach cvs as determined from autumn leaf scar inoculation during three years.

\begin{tabular}{|c|c|c|c|c|c|c|c|c|c|}
\hline \multirow[t]{3}{*}{ Variétés } & \multirow[b]{3}{*}{$\begin{array}{l}\text { Dates } \\
\text { Inoc. }\end{array}$} & \multicolumn{8}{|c|}{ Pourcentages d'inoculations positives } \\
\hline & & \multicolumn{3}{|c|}{60 spores/plaie } & \multicolumn{4}{|c|}{600 spores/plaie } & \multirow{2}{*}{$\begin{array}{l}\text { Moyenne } \\
\text { générale }\end{array}$} \\
\hline & & $\begin{array}{c}18.09 \\
1980\end{array}$ & $\begin{array}{c}18.09 \\
1981\end{array}$ & Moyenne & $\begin{array}{c}27.09 \\
1979\end{array}$ & $\begin{array}{c}18.09 \\
1980\end{array}$ & $\begin{array}{c}18.09 \\
1981\end{array}$ & Moyenne & \\
\hline I.N.R.A. 3689 & & 2 & 12 & 7 & 32 & 34 & 34 & 33,3 & 20,1 \\
\hline J. H. Hale & & 18 & 8 & 13 & 12 & 52 & 28 & 30,7 & 21,8 \\
\hline Harken & & 6 & 12 & 9 & 42 & 36 & 58 & 45,3 & 27,1 \\
\hline Fuzador & & 36 & 2 & 19 & 10 & 62 & 56 & 42,7 & 30,8 \\
\hline Gemfree & & 6 & 8 & 7 & 44 & 70 & 52 & 55,3 & 31,1 \\
\hline Fortyniner & & 16 & 10 & 13 & 46 & 52 & 54 & 50,7 & 31,8 \\
\hline Dixired & & 22 & 14 & 18 & 34 & 58 & 48 & 46,7 & 32,3 \\
\hline Nectared 4 & & 44 & 8 & 26 & 10 & 88 & 44 & 47,3 & 36,6 \\
\hline Halehaven & & 18 & 14 & 16 & 60 & 58 & 60 & 59,3 & 37,6 \\
\hline Troubador & & 26 & 22 & 24 & 32 & 76 & 50 & 52,7 & 38,3 \\
\hline Stark Redgold & & 20 & 20 & 20 & 44 & 78 & 50 & 57,3 & 38,6 \\
\hline Early.O'Henry & & 18 & 16 & 17 & 58 & 66 & 60 & 61,3 & 39,1 \\
\hline Cardinal & & 2 & 14 & 8 & 64 & 78 & 74 & 72,0 & 40,0 \\
\hline Nectared 6 & & 14 & 28 & 21 & 48 & 74 & 60 & 60,7 & 40,8 \\
\hline Everts & & 28 & 26 & 27 & 26 & 82 & 58 & 55,3 & 41,1 \\
\hline Southland & & 26 & 16 & 21 & 30 & 84 & 72 & 62,0 & 41,5 \\
\hline Nerine & & 38 & 42 & 40 & 16 & 56 & 58 & 43,3 & 41,6 \\
\hline Mayfair & & 24 & 18 & 21 & 50 & 84 & 60 & 64,7 & 42,8 \\
\hline Mme Girerd & & 14 & 18 & 16 & 68 & 66 & 78 & 70,7 & 43,3 \\
\hline Moongrand & & 42 & 20 & 31 & 64 & 78 & 52 & 64,7 & 47,8 \\
\hline Frederica & & 40 & 24 & 32 & 42 & 82 & 88 & 70,7 & 51,3 \\
\hline Clio & & 34 & 56 & 45 & 36 & 80 & 66 & 60,7 & 52,8 \\
\hline Vivian & & 46 & 20 & 33 & 68 & 76 & 90 & 78,0 & 55,5 \\
\hline Stark Sunglo & & 46 & 48 & 47 & 24 & 90 & 80 & 64,7 & 55,8 \\
\hline Redhaven 1161 & & 34 & 54 & 44 & 54 & 84 & 82 & 73,3 & 58,6 \\
\hline Shasta & & 70 & 56 & 63 & 30 & 86 & 64 & 60,0 & 61,5 \\
\hline Redhaven 3288 & & 68 & 40 & 54 & 76 & 90 & 60 & 75,3 & 64,6 \\
\hline
\end{tabular}

Analyse statistique des rangs de classement :

- Effet « concentration d'inoculum » hautement significatif

- $\chi_{2}$ calculé $=45,4$,

- $\chi_{2}$ théorique à 0,01 p. $100=13,2$.

- Effet « réceptivité variétale » hautement significatif

- $\chi_{2}$ calculé $=53,4$,

- $\chi_{2}$ théorique à 0,01 p. $100=45,6$. 
TABLEAU 2

Classement des variétés de pêcher par ordre de sensibilité à F. amygdali en fonction de la concentration d'inoculum appliqué sur les plaies pétiolaires.

Classification of peach varieties according to their susceptibility to F. amygdali : susceptibility was determined after inoculation of leaf scars with different concentrations of inoculum.

\begin{tabular}{|c|c|c|c|c|}
\hline Classes & 1 & 2 & 3 & 4 \\
\hline $\begin{array}{l}\text { p. } 100 \text { inoculations } \\
\text { positives }\end{array}$ & $0-20$ & $21-40$ & $41-60$ & $61-80$ \\
\hline 60 spores/plaie & $\begin{array}{l}\text { Stark Redgold } \\
\text { Fuzador } \\
\text { Dixired } \\
\text { Early O'Henry } \\
\text { Mme Girerd } \\
\text { Halehaven } \\
\text { Fortyniner } \\
\text { J. H. Hale } \\
\text { Harken } \\
\text { Cardinal } \\
\text { I.N.R.A. } 3689 \\
\text { Gemfree }\end{array}$ & $\begin{array}{l}\text { Nerine } \\
\text { Vivian } \\
\text { Frederica } \\
\text { Moongrand } \\
\text { Everts } \\
\text { Nectared } 4 \\
\text { Troubador } \\
\text { Mayfair } \\
\text { Southland } \\
\text { Nectared } 6\end{array}$ & $\begin{array}{l}\text { Redhaven } 3288 \\
\text { Stark Sunglo } \\
\text { Clio } \\
\text { Redhaven } 1161\end{array}$ & Shasta \\
\hline 600 spores/plaie & & $\begin{array}{l}\text { I.N.R.A. } 3689 \\
\text { J. H. Hale }\end{array}$ & $\begin{array}{l}\text { Shasta } \\
\text { Halehaven } \\
\text { Stark Redgold } \\
\text { Everts } \\
\text { Gemfree } \\
\text { Troubador } \\
\text { Fortyniner } \\
\text { Nectared } 4 \\
\text { Dixired } \\
\text { Harken } \\
\text { Nerine } \\
\text { Fuzador }\end{array}$ & $\begin{array}{l}\text { Vivian } \\
\text { Redhaven } 3288 \\
\text { Redhaven } 1161 \\
\text { Cardinal } \\
\text { Frederica } \\
\text { Mme Girerd } \\
\text { Stark Sunglo } \\
\text { Moongrand } \\
\text { Mayfair } \\
\text { Southland } \\
\text { Early O'Henry } \\
\text { Nectared } 6 \\
\text { Clio }\end{array}$ \\
\hline
\end{tabular}

La comparaison des résultats obtenus aux 2 concentrations d'inoculum apporte des informations complémentaires sur la sensibilité des variétés à la maladie et permet de mieux analyser les diverses situations susceptibles d'être rencontrées dans la nature. On peut distinguer comme :

- peu sensibles : "J. H. Hale » et «I.N.R.A. 3689 ", qui sont faiblement attaquées aussi bien aux concentrations d'inoculum élevées (classe 2) que faibles (classe 1).

- très sensibles : "Shasta », "Redhaven 3288 », " Redhaven 1161 », «Stark Sunglo » et « Clio », qui sont très attaquées aux fortes doses d'inoculum (classe 4) ainsi qu'aux faibles (classe 3-4).

- sensibles : ayant un comportement intermédiaire, généralement moins touchées aux faibles concentrations d'inoculum qu'aux fortes. Parmi ces variétés dont le classement est plus difficile à caractériser, on observe que certaines, à faible dose, s'avèrent plutôt résistantes (« Halehaven », « Fortyniner », « Harken ») et d'autres, à forte dose, sont très sensibles («Vivian », «Frederica »).

On peut supposer que le classement incertain d'un grand nombre de variétés s'explique par la nature même du test qui s'adresse à des plaies pétiolaires qui ne sont pas au même stade de préparation d'abscission.

\section{B. Sensibilité des bourgeons suivant les variétés}

Malgré l'utilisation de 2 concentrations d'inoculum 1000 fois plus élevées que sur plaies pétiolaires, le taux de pénétration du parasite par les bourgeons aboutissant à un chancre est faible. Pour la concentration d'inoculum la plus élevée (600 000 spores par bourgeon), le pourcentage moyen d'infections chez la variété la plus sensible ne dépasse pas 22 p. 100 et, à la plus faible concentration d'inoculum utilisée (60000 spores par bourgeon), la réceptivité des bourgeons est pratiquement nulle (tabl. 3).

Bien que statistiquement le classement des variétés par ordre de sensibilité soit comparable lors des 3 années d'expérimentation, les différences entre variétés ne sont pas suffisantes pour que l'on attribue à ce classement une signification pratique. L'analyse statistique ne révèle pas de différences de sensibilité entre variétés.

\section{CONCLUSION}

Les différences de sensibilité mises en évidence peuvent expliquer les variations de dégâts dus au Fusicoccum dans la nature en fonction des choix variétaux opérés par les arboriculteurs. En fait, c'est au niveau des plaies pétiolaires que les différences de réceptivité 
TABLEAU 3

Comparaison de la réceptivité des bourgeons de 27 variétés de pêcher à Fusicoccum amygdali par contamination artificielle de printemps sur 3 années.

Susceptibility to peach canker of 27 peach cvs as determined from spring bud inoculation during three years.

\begin{tabular}{|c|c|c|c|c|c|c|c|c|c|}
\hline \multirow[b]{3}{*}{$\begin{array}{c}\text { Dates } \\
\text { Contam. }\end{array}$} & \multicolumn{9}{|c|}{ Pourcentages d'infections positives } \\
\hline & \multicolumn{4}{|c|}{60000 spores/bourgeon } & \multicolumn{4}{|c|}{600000 spores/bourgeon } & \multirow{2}{*}{$\begin{array}{l}\text { Moyenne } \\
\text { générale }\end{array}$} \\
\hline & $\begin{array}{c}18.03 \\
1980\end{array}$ & $\begin{array}{c}17.03 \\
1981\end{array}$ & $\begin{array}{l}5.03 \\
1982\end{array}$ & Moyenne & $\begin{array}{c}18.03 \\
1980\end{array}$ & $\begin{array}{c}17.03 \\
1981\end{array}$ & $\begin{array}{l}5.03 \\
1982\end{array}$ & Moyenne & \\
\hline Everts & 0 & 0 & 2 & 0,7 & 7 & 0 & 8 & 5 & 2,8 \\
\hline Mayfair & 4 & 4 & 4 & 4,0 & 4 & 0 & 4 & 2,7 & 3,3 \\
\hline Early O'Henry & 1 & 0 & 2 & 1,0 & 5 & 2 & 18 & 8,3 & 4,6 \\
\hline Vivian & 4 & 0 & 4 & 2,7 & 4 & 12 & 4 & 6,7 & 4,7 \\
\hline Dixired & 1 & 2 & 2 & 1,7 & 18 & 4 & 4 & 8,7 & 5,2 \\
\hline I.N.R.A. 3689 & 2 & 2 & 10 & 4,7 & $-{ }^{*}$ & - & 6 & 6,0 & 5,3 \\
\hline Gemfree & 2 & 2 & 2 & 2,0 & 4 & 6 & 16 & 8,7 & 5,3 \\
\hline Southland & 5 & 0 & 4 & 3,0 & 5 & 2 & 16 & 7,7 & 5,3 \\
\hline Frederica & 1 & 2 & 10 & 2,1 & 6 & 0 & 14 & 6,7 & 5,5 \\
\hline Nectared & 10 & 2 & 0 & 4,0 & 14 & 4 & 6 & 8,0 & 6,0 \\
\hline Redhaven 3288 & 4 & 0 & 6 & 3,3 & 3 & 4 & 22 & 9,7 & 6,5 \\
\hline Shasta & 1 & 2 & 2 & 1,7 & 22 & 8 & 4 & 11,3 & 6,5 \\
\hline J. H. Hale & 0 & 8 & 2 & 3,3 & 14 & 12 & 4 & 10,0 & 6,6 \\
\hline Harken & 4 & 0 & 4 & 2,7 & 16 & 0 & 20 & 12,0 & 7,3 \\
\hline Cardinal & 7 & 6 & 14 & 9,0 & - $^{*}$ & - & 6 & 6,0 & 7,5 \\
\hline Fuzador & 1 & 2 & 2 & 1,7 & 17 & 2 & 22 & 13,7 & 7,7 \\
\hline Redhaven 1161 & 1 & 0 & 6 & 2,3 & 5 & 16 & 22 & 14,3 & 8,3 \\
\hline Nerine & 0 & 0 & 12 & 4,0 & 11 & 10 & 18 & 13,0 & 8,5 \\
\hline Halehaven & 4 & 2 & 2 & 2,7 & 25 & 14 & 12 & 17,0 & 9,8 \\
\hline Nectared 6 & 8 & 10 & 6 & 8,0 & 6 & 12 & 18 & 12,0 & 10,0 \\
\hline Stark Sunglo & 2 & 0 & 8 & 3,3 & 28 & 12 & 14 & 18,0 & 10,6 \\
\hline Clio & 3 & 0 & 6 & 3,0 & 11 & 8 & 40 & 19,7 & 11,3 \\
\hline Moongrand & 11 & 0 & 2 & 4,3 & 35 & 14 & 12 & 20,3 & 12,3 \\
\hline Fortyniner & 1 & 4 & 6 & 3,7 & 19 & 8 & 40 & 22,3 & 13,0 \\
\hline Stark Redgold & 5 & 2 & 6 & 4,3 & 22 & 10 & 34 & 22,0 & 13,1 \\
\hline
\end{tabular}

* Non contaminés en raison du matériel végétal insuffisant.

Analyse statistique des rangs de classement :

- Effet « concentration d'inoculum » hautement significatif

- $\chi_{2}$ calculé : 56,7 ,

- $\chi_{2}$ théorique à 0,01 p. $100=15,09$.

- Effet « réceptivité variétale » non significatif

- $\chi_{2}$ calculé $=31,4$,

- $\chi_{2}$ théorique a $0,01 \mathrm{p}$. $100=42,9$.

variétale apparaissent avec le plus de netteté. L'apport d'une suspension de spores sur la plaie pétiolaire frấche est la seule méthode d'inoculation à retenir car elle permet d'intervenir à un stade déterminé et est très peu sujette à la présence d'autres microorganismes. A l'inverse, la contamination des bourgeons est plus imprécise : stade de sensibilité très étalé et présence d'autres microorganismes qui peuvent gêner l'installation du parasite. Ainsi, dans nos conditions d'expérimentation, la contamination des bourgeons ne permet pas de cribler les variétés pour leur résistance à F. amygdali.

Les résultats obtenus doivent inciter à une surveillance toute particulière des vergers placés dans les régions les plus favorables à la maladie du chancre (Sud-Ouest), coplantés en variétés très sensibles comme "Shasta ", "Redhaven 3288 ", "Redhaven 1161 », "Stark Sunglo » ou "Clio ». Pour les nouvelles plantations dans de telles régions, les arboriculteurs soucieux de ne pas être confrontés à la maladie avec trop de gravité, peuvent choisir les moins sensibles si celles-ci sont susceptibles de leur apporter satisfaction au plan agronomique ou économique.

Dans les régions exposées à une pression de maladie traditionnellement modérée (Sud-Est), le choix à retenir est plus large car une proportion non négligeable des 27 variétés que nous avons mises en comparaison s'avère douée d'une certaine résistance en présence de faibles concentrations d'inoculum (60 spores/plaie). Sont à 'classer parmi ces variétés : " Halehaven », "Fortyniner ", " Harken » mais aussi " Fuzador", "Dixired». En ce qui concerne la concentration d'inoculum, le nombre de 600 spores par plaie peut paraître élevé mais il ne faut pas oublier que les spores de $F$. amygdali sont agglomérées dans des cirrhes et sont disséminées par paquets, d'où une concentration qui peut être très forte.

Outre la connaissance sur la sensibilité au Fusicoccum des variétés déjà commercialisées, ce travail montre qu'il est déterminant de préciser, en verger d'expérimentation, la sensibilité des nouvelles variétés et, dans l'avenir, qu'il mérite d'être entrepris avant toute nouvelle diffusion, comparativement aux variétés de référence : «I.N.R.A. 3689 », « Redhaven 3288 », « Halehaven ». 


\section{RÉFÉRENCES BIBLIOGRAPHIQUES}

Grosclaude C., 1956. Recherches sur le chancre du pêcher causé par Fusicoccum amygdali Del. Ann. Epiphyt., 3, 397-419.

Jailloux F., Froidefond G., 1974. Recherche d'un test de sensibilité variétale du pêcher à l'égard du Fusicoccum amygdali Del. Rev. Zool. Agric. Pathol. Vég., 73, 140-148.

Jailloux F., Froidefond G., 1976. Comportement variétal du pêcher à l'égard du Fusicoccum amygdali Del. Résultats 1974-1975. Pomol. Fr., XVII (3), 39-40.
Jailloux F., Froidefond G., 1978a. Comportement variétal du pêcher à l'égard du Fusicoccum amygdali Del. Résultats 1974-1975, 19761977. Arboric. Fruit., 292, 39-41.

Jailloux F., Froidefond G., 1978b. Influence de la concentration de l'inoculum et du stade de défeuillaison sur la réceptivité des plaies pétiolaires du pêcher à l'égard du Fusicoccum amygdali Del., agent du chancre. Ann. Phytopathol., 10 (1), 39-44.

Payghami E., 1978. Etudes anatomiques et histologiques de deux rameaux de pêcher inégalement sensibles au Fusicoccum amygdali Del., agent du chancre. C.R. Acad. Agric. Fr., 64 (11), 872-875. 